\title{
Approaches to Reducing Radiation Dose from Radionuclide Myocardial Perfusion Imaging
}

\author{
Sharmila Dorbala, Ron Blankstein, Hicham Skali, Mi-Ae Park, Jolene Fantony, Charles Mauceri, James Semer, \\ Stephen C. Moore, and Marcelo F. Di Carli \\ Division of Nuclear Medicine and Molecular Imaging, Department of Radiology, the Noninvasive Cardiovascular Imaging Program, \\ Departments of Medicine (Cardiology) and Radiology, Brigham and Women's Hospital, Boston, Massachusetts
}

Learning Objectives: On successful completion of this activity, participants should be able to (1) describe the application of appropriate use criteria for SPECT and PET myocardial perfusion imaging, (2) apply stress-only/stress-first myocardial perfusion imaging in clinical practice, and (3) list methods to perform lowradiation-dose myocardial perfusion imaging using traditional and novel technology.

Financial Disclosure: Dr. Dorbala was supported by NIH-NHLBI K23HL092299, has received a research grant from Astellas Pharma (unrelated to this work), and has investment interest with General Electric. The authors of this article have indicated no other relevant relationships that could be perceived as a real or apparent conflict of interest.

CME Credit: SNMMI is accredited by the Accreditation Council for Continuing Medical Education (ACCME) to sponsor continuing education for physicians. SNMMI designates each JNM continuing education article for a maximum of 2.0 AMA PRA Category 1 Credits. Physicians should claim only credit commensurate with the extent of their participation in the activity. For CE credit, SAM, and other credit types, participants can access this activity through the SNMMI website (http://www.snmmilearningcenter.org) through April 2018.

Radionuclide myocardial perfusion imaging (MPI) plays a vital role in the evaluation and management of patients with coronary artery disease. However, because of a steep growth in MPI in the mid 2000s, concerns about inappropriate use of MPI and imaging-related radiation exposure increased. In response, the professional societies developed appropriate-use criteria for MPI. Simultaneously, novel technology, image-reconstruction software for traditional scanners, and dedicated cardiac scanners emerged and facilitated the performance of MPI with low-dose and ultra-low-dose radiotracers. This paper provides a practical approach to performing low-radiationdose MPI using traditional and novel technologies.

Key Words: SPECT; low dose; PET; radiation dose; myocardial perfusion imaging; quality assurance

J Nucl Med 2015; 56:592-599

DOI: 10.2967/jnumed.112.115097

$\mathbf{O}$ ver the past $40 \mathrm{y}$, radionuclide myocardial perfusion imaging (MPI) has become a major tool in the noninvasive evaluation of coronary artery disease (CAD). During that time, advances in MPI technology and radiotracers have increased opportunities for improved diagnosis and treatment of patients with CAD. However, there were concerns about its overutilization (1), especially in lower-risk patients, which had coincided with a 6-fold increase in background radiation from medical imaging (2). In response, multiple professional societies have jointly developed appropriate use criteria (AUC) to encourage

Received Jan. 2, 2015; revision accepted Feb. 27, 2015.

For correspondence or reprints contact: Sharmila Dorbala, Brigham and Women's Hospital, 70 Francis St., Shapiro 5th Floor, Room 128, Boston, MA 02115.

E-mail: sdorbala@partners.org

Published online Mar. 12, 2015.

COPYRIGHT (c) 2015 by the Society of Nuclear Medicine and Molecular Imaging, Inc. the appropriate use of MPI (3) and to lower the costs of and radiation dose from MPI. Manufacturers of nuclear medicine equipment have also responded to radiation concerns by introducing technologic advances that allow individualized low-dose protocols while maintaining or enhancing image quality, thereby paving the way for important changes in the practice of MPI. This paper will focus on the vital importance of appropriate patient selection, patient-centered techniques to reduce radiation dose, and practical ways to reduce the lifetime radiation dose for the individual patient as well as for the population of patients being considered for MPI.

\section{THE VITAL IMPORTANCE OF RADIONUCLIDE MPI TO MANAGE CAD}

Radionuclide MPI is the most mature cardiovascular imaging technique, with advanced quantitative tools and a vast evidence base in over 100,000 patients (4). Stress MPI with SPECT and PET is widely used to identify the hemodynamic significance of CAD. The greatest strength of MPI, however, is its established value for risk assessment (5). The extent and severity of ischemia and scarring on SPECT and PET MPI are powerful predictors of future cardiovascular events (5). In addition, left ventricular ejection fraction measured on SPECT and PET MPI has well-established incremental value for patient management and risk stratification (5). MPI is costeffective for the management of CAD. In patients with stable angina pectoris, a noninvasive SPECT MPI-guided management strategy has been shown to be economically superior to an anatomic approach guided by invasive coronary angiography without significant differences in clinical outcomes (6). More recently, radionuclide imaging of myocardial blood flow with PET (and SPECT) has been shown to be an indispensable tool for the evaluation and management of CAD $(7,8)$. Furthermore, several recent advances in PET and SPECT hardware and software facilitate rapid, high-count imaging, and low-dose imaging $(9,10)$. With these unique capabilities, the clinical benefits of an appropriately performed MPI study are indisputable. 


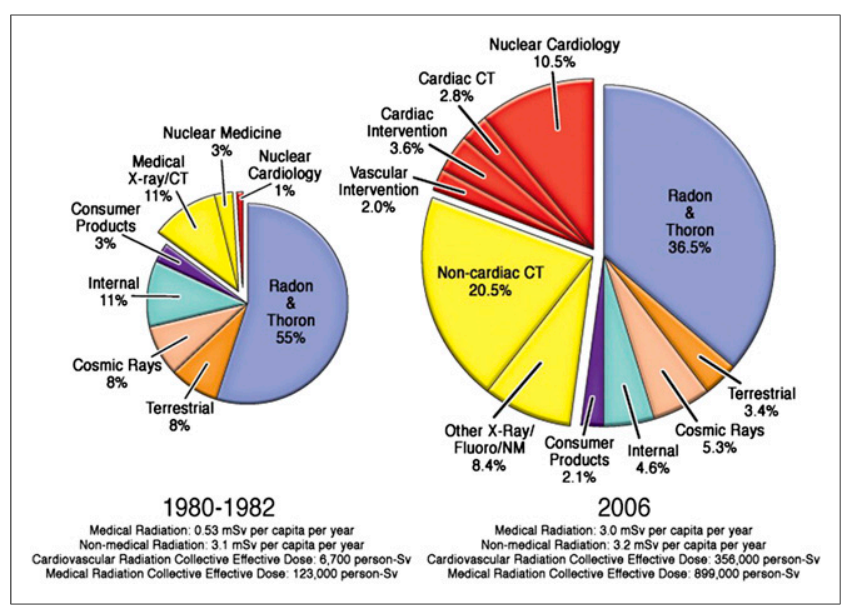

FIGURE 1. Increasing radiation burden in United States and contributions from medical imaging. Collective dose from medical imaging increased 6fold in 2006 compared with early 1980s. (Reprinted with permission of (2).)

\section{THE NEED TO REDUCE RADIATION DOSE FROM MPI}

The primary concern about ionizing radiation from MPI relates to the stochastic effects and potential risk of the development of cancer decades later (11). Cancer risk is estimated on the basis of linear downward extrapolation of data from atom bomb survivors to the ranges of medical imagingrelated radiation (11). The risk from a medical imaging-related low level of radiation dose is small and difficult to estimate accurately $(2,11)$. However, the overall radiation burden to the U.S. population doubled from the early 1980 s to 2006 . Although in the early 1980s medical imaging accounted for $15 \%$ of the U.S. population's per capita exposure to ionizing radiation from all sources $(0.54$ of $3.6 \mathrm{mSv}$ ), in $200648 \%$ of per capita exposure ( 3 of $6.255 \mathrm{mSv}$ ) came from medical imaging (12). Furthermore, the contributions of nuclear cardiology procedures to ionizing radiation increased 10-fold over this period (Fig. 1) (2). A standard rest-stress ${ }^{99 \mathrm{~m} T c}$ MPI study can be performed with a dose of about $12 \mathrm{mSv}$ (13), compared with an average natural background radiation dose in the United States of $3 \mathrm{mSv}$. The radiation dose from MPI is declining significantly with new technologies. No data exist to relate an increased risk of cancer from ionizing radiation at these levels (2). But the performance of millions of procedures has raised concerns over increasing radiation dose to the population and the consequent minimal risk of future cancer related to radiation from diagnostic imaging (12).

Efforts to reduce radiation dose from MPI are important, as long as the concomitantly increased level of image noise does not significantly compromise the ability of physicians to have confidence in making the correct diagnoses based on the images. Also, the risk of performing MPI must be balanced against the risk of missed or delayed diagnosis and treatment from not performing MPI. Radiation dose-reduction strategies and education may promote the safe and effective use of MPI. Informed patients and physicians may be more accepting of MPI, allowing more patients to benefit from this technology. Indeed, a focus on reducing radiation dose from imaging may compel us to more closely scrutinize the need for MPI and reduce the volume of unnecessary tests, thereby reducing lifetime cumulative radiation dose to the patient.

\section{RADIATION DOSE REDUCTION BEFORE MPI: APPROPRIATE USE}

Radiation dose from medical imaging can be reduced before, at the time of, or after completion of the test (Fig. 2). One of the main ways to reduce radiation dose before the performance of a test is to avoid tests that are not needed.

Over the last decade, several professional societies have developed AUC for various diagnostic tests and therapeutic procedures. Appropriate use is defined, by the RAND Corp., as an indication wherein the expected clinical benefit of the test outweighs the risks of the procedure. AUC for cardiac radionuclide imaging, first published in 2005, were most recently updated in 2009 (3). Indications for MPI were categorized on the basis of the median scores of 15 expert panelists. A range of $1-3$ is considered rarely appropriate (also previously referred to as inappropriate), 4-7 may be appropriate (also previously referred to as uncertain), and 7-9 appropriate. Of a list of 67 indications for MPI, 9 were considered uncertain, 25 inappropriate, and the rest appropriate (3). Of note, the radionuclide AUC did not distinguish the indications for SPECT from PET MPI; the expert rating applied equally to SPECT and PET. The value of AUC in reducing radiation dose is obtained from avoiding the tests that may be rarely appropriate. When imaging is appropriate, the benefit of an optimal test generally outweighs its potential risk. The appropriateness of any given indication for MPI can be checked online using a radionuclide AUC app (https://itunes.apple.com/us/app/appropriate-use-criteria-auc/ id391068250? $\mathrm{mt}=8$; Astellas Pharma US).

The AUC methodology has certain limitations. AUC were developed on the basis of expert opinion and expert interpretation of existing clinical and trial evidence, whereas guidelines are developed on the basis of clinical trial evidence. They do not include a list of all possible clinical scenarios. They are not directly based on the frequency with which the test reclassifies the extent or severity of a patient's disease or directly affects the choice of treatment. Also, they do not take into account the costs of the test, cost effectiveness, relative performance of one test versus alternative tests, ionizing radiation, or the impact of repeat testing or layered testing.

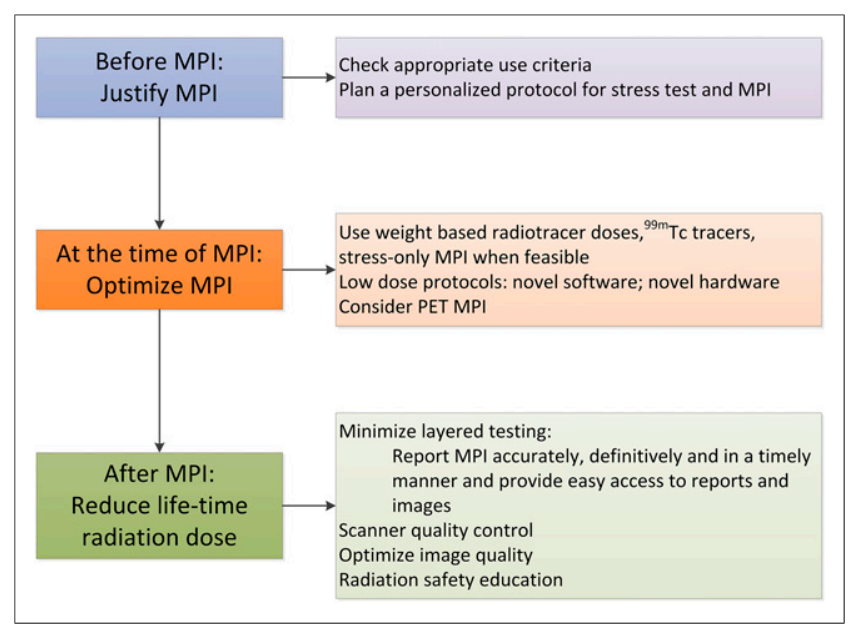

FIGURE 2. Practical ways to implement reduced-radiation-dose MPI program. 
TABLE 1

Five Things Physicians and Patients Need to Know $(15,16)$

\begin{tabular}{ll}
\hline Source & Point \\
\hline SNMMI & Do not perform routine annual stress testing after coronary artery revascularization. \\
ASNC & $\begin{array}{l}\text { Do not perform stress cardiac imaging or coronary angiography in patients without cardiac symptoms } \\
\text { unless high-risk markers are present. }\end{array}$ \\
Do not perform cardiac imaging in patients who are at low risk. & Do not perform radionuclide imaging as part of routine follow-up in asymptomatic patients. \\
& $\begin{array}{l}\text { Do not perform cardiac imaging as preoperative assessment in patients scheduled to undergo low- or } \\
\text { intermediate-risk noncardiac surgery. } \\
\text { Use methods to reduce radiation exposure in cardiac imaging whenever possible, including not performing such } \\
\text { tests when the benefits will likely be limited. }\end{array}$ \\
\hline
\end{tabular}

\section{Frequency of Appropriate MPI per AUC}

The frequency with which MPI is used appropriately may vary from region to region and in different practices. In one study (14), from 6 sites in the UnitedHealthcare system, most of the MPI studies were performed for appropriate indications (85.6\%) and only $14.4 \%$ were performed for inappropriate indications (rarely appropriate). Inappropriate studies (or rarely appropriate) were more common in asymptomatic individuals, women, and preoperative patients (14). The American Society of Nuclear Cardiology (ASNC) incorporated some of these indications into the Choosing Wisely campaign to reduce inappropriate use of MPI. Choosing Wisely is an effort by the American Board of Internal Medicine partnering with Consumer Reports and in collaboration with several medical societies (including the Society of Nuclear Medicine and Molecular Imaging [SNMMI], ASNC, and others), developed to curb the growth in the use of unnecessary imaging tests. It uses the philosophy of "5 things that physicians and patients should question." Table 1 lists the SNMMI and ASNC Choosing Wisely points related to MPI $(15,16)$.

\section{Methods to Increase the Appropriate Use of MPI}

Appropriate use of MPI can be increased only by improving knowledge about AUC for both referring provider and imaging provider. Unfortunately, educational efforts to reduce the rates of rarely appropriate studies have had mixed results. Initial short-term declines in the proportion of rarely appropriate tests ordered were not sustained over the long term (17).

The appropriateness of MPI can be tracked at the time of order entry using one of several online tools. The American College of
Cardiology FOCUS (Formation of Optimal Cardiovascular Use Strategies) is a web-based quality improvement tool developed to track and improve the appropriate use of MPI. In a preliminary analysis, the proportion of rarely appropriate indications decreased from $10 \%$ to $5 \%$ for the sites participating in the FOCUS PIM (practice improvement module) (18). Also, decision support tools (DST), incorporated into physician electronic-order-entry systems, can guide the referring physicians through steps to ensure appropriate use of the test. One such system, the AUC-DST, showed that the frequency of appropriate tests increased from $49 \%$ to $61 \%$ and the frequency of rarely appropriate tests decreased from $22 \%$ to $6 \% 8 \mathrm{mo}$ after the implementation of the AUC-DST (19). Ideally, the referring physician should ensure the appropriateness of the test at the time of order entry using clinical DST. Indeed, it has been mandated by law that documentation of AUC using DST for ordering advanced imaging tests (including SPECT and PET MPI) will, by January 2017, be a prerequisite to receiving payment for imaging services for Medicare patients $(20,21)$. This measure likely will increase the rate of appropriate studies.

Because cardiovascular imaging has become fairly complex, choosing a suitable test from the many possible tests can be challenging for ordering physicians who may not be imaging experts. In complex cases, a discussion between the referring physicians and imaging physicians, who have expertise in multimodality imaging, can ensure that the most appropriate test is selected for a given indication and patient. In some cases, the most appropriate test may be a nonimaging exercise treadmill test, stress echocardiography, or MR imaging-based MPI. This discussion is facilitated by a systematic

TABLE 2

Steps to Create Protocol Plan for MPI

\begin{tabular}{ll}
\hline Step & Review electronic medical records to define clinical question. \\
\hline 1 & $\begin{array}{c}\text { Check for recently performed cardiac evaluations to avoid duplicate testing and layered testing for similar clinical } \\
\text { symptoms. }\end{array}$ \\
\hline 3 & $\begin{array}{l}\text { If clinical question is not clear or if test ordered is not the most appropriate test, discuss with referring physician for } \\
\text { clarification. }\end{array}$ \\
\hline 5 & Plan appropriate stress technique. \\
\hline
\end{tabular}


review (a "protocol plan") of the patient's history (Table 2), at least $1 \mathrm{~d}$ before the MPI, to change the test if needed and to contact the patient. Imaging laboratory personnel, fellows in training, or staff members experienced in imaging can prepare a protocol plan for the studies.

The appropriate use of MPI is expected eventually to reduce cumulative lifetime radiation exposure from imaging of patients with CAD, although this has not yet been studied directly on a large scale.

\section{RADIATION DOSE REDUCTION DURING THE TEST: OPTIMIZE MPI}

SNMMI and ASNC recommend a patient-centered imaging approach taking into account radiation dose. ASNC issued an information statement on reducing radiation exposure in MPI and recommended several methods that, if used appropriately, can reduce radiation dose to $9 \mathrm{mSv}$ or less in $50 \%$ of patients referred for SPECT or PET MPI studies (22). Selection of protocols for MPI should also take into account the clinical question, the image quality, potential risks incurred from future testing stemming from the results of MPI and from radiation, costs, patient convenience, and assurances that the patient and staff are receiving the lowest possible radiotracer dose (23). However, image quality should not be significantly jeopardized by dose reduction. Patient characteristics such as body habitus, claustrophobia, ability to lie supine for imaging, and stress modality are also important considerations. Appropriate selection of radiotracer, the use of novel imaging protocols, and, when available, the use of novel reconstruction methods, hardware, collimators, and software are critical to reducing patient radiation dose.

\section{Selection of Radiotracers}

Estimated whole-body effective radiation dose (averaged over various organs and averaged for men and women) is directly related to the half-life of the radiotracer and dose of radiotracer administered (Table 3 ).
For SPECT MPI, ${ }^{99 \mathrm{~m}} \mathrm{Tc}$ agents are preferred over ${ }^{201} \mathrm{Tl}$ because of their shorter half-life, significantly lower effective dose, and superior image quality. For PET MPI, ${ }^{82} \mathrm{Rb}$ and ${ }^{13} \mathrm{~N}$-ammonia, when available, offer an even greater reduction in radiation dose compared with ${ }^{99 \mathrm{~m}} \mathrm{Tc}$ SPECT. Radiation dose from PET MPI can be further lowered with imaging in 3-dimensional (3D) mode, as this mode offers much higher-count imaging and permits half-dose imaging, which may be especially useful in children. The mean estimated whole-body effective dose from SPECT and PET perfusion tracers is listed in Table 3.

For SPECT and PET MPI, a weight- or body mass index-based adjusted radiotracer dose may be better than a fixed dose for all, to balance low radiation dose with optimal image quality (24). Indeed, Marcassa et al. (25) recently documented a 58\% radiation dose savings to patients, and a $50 \%$ dose reduction to cardiologists performing the test, by switching from a fixed-dose protocol to a weight-based ${ }^{99 \mathrm{~m}} \mathrm{Tc}$ dosing protocol and finally to low-dose MPI using novel software-based reconstructions. A sample table of weight-based ${ }^{99 \mathrm{~m}} \mathrm{Tc}$ dosing and estimated whole-body effective dose is included in Supplemental Table 1. Radiation dose from MPI can be additionally reduced using any of the protocols and technologies described below.

\section{Stress-First or Stress-Only Imaging Protocols for Reduced-Dose MPI}

Stress-first or stress-only MPI, as well as low-radiotracer-dose protocols (half-dose or less than half-dose) using novel scanners, collimators, or software, can significantly reduce radiation dose from SPECT MPI compared with standard-dose rest-stress MPI protocols. Stress-first SPECT imaging has several other advantages (Supplemental Table 2), and although supported by the imaging societies for over a decade now, the stress-first protocol has not been widely implemented because of several challenges (Supplemental Table 2). But a decline in the frequency of abnormal scan results from $41 \%$ in 1991 to $8.7 \%$ in 2009 (26) combined with the soaring costs of medical imaging ( $>6$ million SPECT MPI studies per year) (2) makes a strong case for stress-only imaging.

TABLE 3

Estimation of Effective Radiation Dose from Various Myocardial Perfusion Radiotracers (13)

\begin{tabular}{|c|c|c|c|c|c|c|c|}
\hline \multirow[b]{3}{*}{ Radiopharmaceutical } & \multirow{3}{*}{$\begin{array}{l}\text { Effective dose } \\
\text { (mSv/MBq) }\end{array}$} & \multicolumn{4}{|c|}{ Administered activity } & & \\
\hline & & \multicolumn{2}{|c|}{$\mathrm{MBq}$} & \multicolumn{2}{|c|}{$\mathrm{mCi}$} & \multicolumn{2}{|c|}{ Estimated dose (mSv) } \\
\hline & & Full dose & Half dose & Full dose & Half dose & Full-dose study & Half-dose study \\
\hline${ }^{82} \mathrm{Rb}$ rest or stress & 0.0017 & 1,480 & 740 & 40 & 20 & 2.52 & 1.26 \\
\hline${ }^{13} \mathrm{~N}$-ammonia rest or stress & 0.0027 & 740 & 370 & 20 & 10 & 2.0 & 1.0 \\
\hline 99mTc-sestamibi rest & 0.0079 & 296 & 148 & 8 & 4 & 2.34 & 1.17 \\
\hline 99mTc-sestamibi stress & 0.009 & 888 & 444 & 24 & 12 & 8.0 & 4.0 \\
\hline 99mTc-tetrofosmin rest & 0.0069 & 296 & 148 & 8 & 4 & 2.0 & 1.0 \\
\hline${ }^{99 m}$ Tc-tetrofosmin stress & 0.0069 & 888 & 444 & 24 & 12 & 6.13 & 3.1 \\
\hline${ }^{201} \mathrm{Tl}$ & 0.14 & 148 & 74 & 4 & 2 & 20.72 & 10.36 \\
\hline
\end{tabular}

Recommended MPI radiotracer doses for conventional scanners are 8-12 mCi of 99mTc-sestamibi for rest imaging and 24-36 mCi for stress imaging, $40-60 \mathrm{mCi}$ of ${ }^{82} \mathrm{Rb}$ for $2 \mathrm{D}$ imaging and $20 \mathrm{mCi}$ for $3 \mathrm{D}$ imaging (58), $20 \mathrm{mCi}$ of ${ }^{13} \mathrm{~N}$-ammonia for $2 \mathrm{D}$ imaging and $10 \mathrm{mCi}$ for $3 \mathrm{D}$ imaging (58), and 2.5-4 mCi for ${ }^{201} \mathrm{Tl}$ imaging (59) $(1 \mathrm{mCi}=37 \mathrm{MBq})$. New estimates of ${ }^{82} \mathrm{Rb}$ dose are significantly lower (0.00126 mSv/ $\mathrm{MBq}$ ) (60). Full-dose PET radiotracer is used for 2D imaging and half-dose for 3D imaging; typically, equal dose of radiotracer is administered for rest and for stress PET MPI. Average activities are listed. Estimated dose is effective dose multiplied by administered activity. Dose is calculated for rest and stress scans separately, and if attenuation correction is used, $0.3-0.7 \mathrm{mSv}$ is added for CT and 0.3 $\mathrm{mSv}$ for radionuclide transmission scanning (13). 
A growing body of literature supports the utility of stress-first SPECT imaging (24,27-34). If the stress MPI results are normal, the rest scan can be avoided, with significant savings in cost, time, and radiotracer exposure to the patient (35\% dose reduction) and to the laboratory staff (40\% dose reduction) (35). Stress-first and stress-only PET MPI have not been as widely studied as stress-first and stress-only SPECT MPI.

Implementation of Stress-First MPI. A successful implementation of stress-only or stress-first protocols requires careful screening of patients ahead of the test. Experienced imaging physicians must be available to finalize the MPI report, definitively and unequivocally, before discharging the patient from the laboratory. A stress-only study is considered to have normal results when the myocardial perfusion is homogeneous and the ejection fraction and left ventricular volumes are normal during maximal stress, with no ischemic electrocardiogram changes (27). However, if the stress-first results are not normal, a rest scan can be completed on the same day with 3 times the stress radiotracer dose.

Patient Selection for Stress-First MPI. Patient selection is critical for a successful stress-first program. Patients without a prior history of myocardial infarction and an intermediate pretest likelihood of CAD are well suited for stress-first MPI. The pretest likelihood of CAD is calculated using the age, sex, and chest-pain characteristics (Supplemental Table 3). A stress-first imaging protocol is feasible with either exercise or pharmacologic stress and may be used in diabetic patients (27) as well as in morbidly obese patients (30). Patients with a low pretest likelihood of CAD, however, may be considered for treadmill testing alone without imaging. Even among women with an intermediate pretest likelihood of CAD and able to exercise on a treadmill, the WOMEN study (What is the Optimal Method for Ischemia Evaluation in Women?) demonstrated that an initial diagnostic strategy of exercise treadmill testing with imaging, when compared with exercise treadmill testing alone, did not provide any incremental diagnostic or prognostic benefit and reduced downstream costs (36).

Attenuation Correction for Stress-First SPECT MPI. Accurate identification and correction of attenuation artifacts is important in stress-first imaging algorithms to avoid interpretation of attenuation artifacts as real perfusion defects. Although gated SPECT is not helpful in identifying attenuation artifacts (because ischemic wall motion abnormalities typically resolve by the time of image acquisition), prone imaging can be used for troubleshooting fixed inferior-wall perfusion defects. Attenuation correction, however, is the most direct and effective method for correcting attenuation artifacts.

Attenuation correction using radionuclide or CT-based transmission scans significantly reduced the need for rest MPI imaging in as many as $37 \%-48 \%$ of patients scheduled for stress-first SPECT imaging $(31,37)$. Indeed, the primary application of cardiac CT with SPECT and PET MPI is for attenuation correction, with a radiation dose ranging from 0.3 to $1.3 \mathrm{mSv}$ with appropriate cardiac CT dose reduction methods (Supplemental Table 4) (13). The use of attenuationcorrected MPI with gated SPECT is essential for the best clinical application of stress-first or stress-only imaging.

Prognostic Value of Stress-Only Imaging. The excellent prognostic value of a normal stress-only SPECT result from conventional scanners is well established (Supplemental Table 5) (27). In a pooled analysis including 10,438 patients with stress-only MPI, a normal result was associated with an annual event rate of $0.7 \%$, with a relative risk comparable to a normal rest-stress MPI result (5). This area has not been examined for PET MPI.

\section{Novel Reconstruction Software, Scanners, and Collimators for MPI}

Several recent advances in cardiac SPECT software, novel semiconductor detector solid-state SPECT scanners (cadmium zinc telluride [Spectrum Dynamics or GE Healthcare] or thalliumactivated cesium iodide [CsI(Tl)] [Digirad]), and novel collimator design (38) have substantially improved image resolution and lowered radiation dose for MPI $(10,39)$.

Novel iterative reconstruction methods (Astonish [Phillips], wide-beam reconstruction [UltraSPECT Inc.], Flash 3D [Siemens], n-SPEED [Digirad], and Evolution [GE Healthcare]) with resolution recovery and noise reduction provide higher image contrast (with sharper defects and borders) and significantly improve image quality, particularly for low-count imaging studies from halfand quarter-dose radiotracer protocols (40). Despite excellent image quality with shorter imaging times (41-44), not many studies have prospectively evaluated half-dose MPI with novel software. DePuey et al. (42) used half-dose ${ }^{99 \mathrm{~m}} \mathrm{Tc}$ MPI and showed that low-dose MPI with conventional scanners using novel software (wide-beam reconstruction) provides good to excellent image quality in $93 \%$ of patients. The value of the novel software is that existing scanners can be upgraded with advanced software to reduce radiation dose, a much smaller capital investment than buying a new scanner.

Novel solid-state SPECT scanners offer a severalfold increased count sensitivity compared with the conventional $\mathrm{NaI}(\mathrm{Tl})$ scanners because they use cardiofocal imaging and either large parallelhole or multiple-pinhole collimators $(9,10,45)$. Also, attenuation correction is available for some of these scanners (Digirad and GE Healthcare). Iterative-reconstruction protocols combined with resolution recovery and noise reduction are standard for the novel scanners. Each of these enhancements enables low-dose and ultralow-dose MPI.

Because of high count sensitivity, the novel scanners offer significant flexibility with imaging protocols. The initial focus of the novel high-sensitivity scanners was on rapid imaging (2- to 4min imaging times), which is well suited for imaging patients with multiple comorbidities who may not otherwise tolerate longer acquisition times. However, low-radiation-dose, high-quality imaging is the current focus of the novel scanners (Table 4). Some protocols use a half-dose or less (single-day, 111-185 MBq [3-5 $\mathrm{mCi}] / 333-555 \mathrm{MBq}$ [9-15 $\mathrm{mCi}]$ of ${ }^{99 \mathrm{~m}} \mathrm{Tc}$ ) with imaging times of 8 and $6 \mathrm{~min}$ or longer for count-based imaging. The effective radiation dose from stress-only protocols with novel scanners is less than $2 \mathrm{mSv}$. However, enthusiasm for further dose reduction is tempered by the longer acquisition times, which may increase the likelihood of patient motion, especially if the acquisition duration is $7 \mathrm{~min}$ or more.

In multicenter studies, rapid scanning with dedicated cardiac SPECT scanners provided comparable or superior image quality, with a much shorter scan duration than for standard-time scanning with conventional scanners $(46,47)$. However, only one study directly compared low-dose dedicated cardiac SPECT scanning with conventional-dose scanning in the same patients, and one other study simulated low-radiation-dose rest and stress imaging. Einstein et al. (48) directly compared rest ultra-low-dose ${ }^{99 \mathrm{~m}} \mathrm{Tc}$ dedicated cardiac SPECT imaging (133.96 MBq/3.62 mCi) with standarddose conventional SPECT in 110 patients (mean body mass index, $26.1 \pm 2.8 \mathrm{~kg} / \mathrm{m}^{2}$; range, $17.1-30.9 \mathrm{~kg} / \mathrm{m}^{2}$; mean acquisition time range, $9.7-15.2 \mathrm{~min}$ ) from 3 sites and showed comparable image quality with a very low radiation dose when using the dedicated cardiac SPECT scanner $(1.15 \pm 0.24 \mathrm{mSv})$. Nakazato et al. (49) 
TABLE 4

Radiation Dose from Low-Dose Protocols for Novel SPECT Scanners

\begin{tabular}{|c|c|c|c|c|c|c|c|}
\hline Study & Rest dose* & Stress dose ${ }^{\star}$ & $\begin{array}{c}\text { No. of } \\
\text { patients }\end{array}$ & Patient size & Radiotracer & Protocol (1 d) & $\begin{array}{l}\text { Study radiation } \\
\text { dose (mSv) }\end{array}$ \\
\hline \multirow[t]{3}{*}{24} & $296-481(8-13)$ & $\begin{array}{c}462.5 ; 925-1,332 \\
\quad(12.5 ; 25-36)\end{array}$ & 717 & $<91$ kg (200 lb) & 99mTc-sestamibi & LD stress only & 4.2 \\
\hline & & & & & & HD stress only & 8.0 \\
\hline & & & & & & Stress-rest & 11.8 \\
\hline 61 & $185(5)$ & $555(15)$ & 131 & $\mathrm{BMI},<35$ & 99mTc-sestamibi & Rest-stress & 5.8 \\
\hline 62 & $640(17.29)$ & $320(8.65)$ & 50 & BMI, 19-32 & 99mTc-tetrofosmin & Stress-rest & $\begin{array}{l}\text { Stress only, 2.21; } \\
\text { stress + rest, } 6.62\end{array}$ \\
\hline 52 & $185-222(5-6)$ & $370-444(10-12)$ & 137 & BMI, $39 \pm 7$ & 99mTc-tetrofosmin & Stress-rest & $5.10-6.12$ \\
\hline 51 & $222(6)$ & $740(20)$ & 285 & BMI, $29 \pm 5$ & 99mTc-tetrofosmin & Rest-stress & Rest, 1.4; stress, 4.6 \\
\hline 48 & $129.5(3.5)$ & NA & 101 & BMI, 17.1-30.9 & 99mTc-sestamibi & Rest only & 1.2 \\
\hline $\begin{array}{l}{ }^{*} \text { Data a } \\
\text { LD = Ic } \\
\text { Variabl } \\
\text { Discove }\end{array}$ & $\begin{array}{l}\text { egabecquerels fo } \\
\text { ose; HD = high d } \\
\text { an times were use } \\
\text { IM530c (GE Healt }\end{array}$ & $\begin{array}{l}\text { ed by millicuries in } \\
\text { BMI = body mass } \\
\text { count-based acqu } \\
\text { e) }(24,51,52,61) \text {. }\end{array}$ & $\begin{array}{l}\text { ntheses }(r \\
x\left(\mathrm{~kg} / \mathrm{m}^{2}\right. \\
\text { n. The sca }\end{array}$ & $\begin{array}{l}\text { or mean). } \\
\text { e, mean } \pm S D \text {, or } \\
\text { was a Discovery }\end{array}$ & $\begin{array}{l}\text { er limit); NA = not } \\
\text { CT 570c (GE Healtl }\end{array}$ & $\begin{array}{l}\text { cable. } \\
\text { (62), D-SPECT }\end{array}$ & trum Dynamics) (48), c \\
\hline
\end{tabular}

recently simulated low-dose rest and stress SPECT MPI in 79 patients (mean body mass index, $30.0 \pm 6.6$; range, 20.2-54.0 $\mathrm{kg} / \mathrm{m}^{2}$ ) and showed that image quality is adequate even with very low-count images $(\sim 1$ million) and comparable to standard fullcount images. Finally, low-dose ${ }^{99 \mathrm{~m}} \mathrm{Tc}$ MPI with dedicated cardiac scanners is accurate for detecting obstructive CAD on invasive angiography (e.g., in one study visual analysis sensitivity was $92 \%$, specificity $56 \%$, and normalcy 98\%) (50-52). Although the novel scanners offer significant advantages and high-quality imaging with a low radiation dose, they are expensive, at this time, with clinical imaging applications limited to cardiac imaging. An algorithm for reduced-dose SPECT MPI is shown in Figure 3.

\section{Dose Reduction with PET}

Most current-generation PET scanners image in 3D mode and are equipped with advanced hardware and software capabilities for high-resolution, low-dose imaging (9). With time-of-flight, high-definition iterative reconstruction and motion-frozen imaging, an effective spatial resolution of as low as $2 \mathrm{~mm}$ can be achieved with PET MPI (10). When combined with the low dose from PET tracers, PET MPI offers a significantly lower radiation dose than SPECT MPI. Stress-only imaging and low-dose CT imaging are

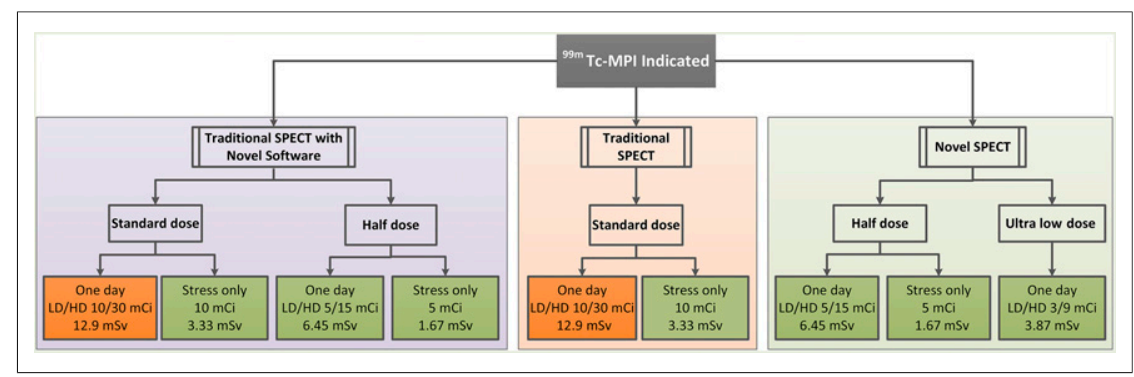

FIGURE 3. Patient-centered protocols for low-radiation-dose MPI: traditional SPECT (orange), traditional SPECT with novel software (purple), and novel SPECT scanners (green). Most MPI procedures that use novel protocols or novel technologies provide $<9-\mathrm{mSv}$ radiation dose from rest-stress ${ }^{99 \mathrm{~m} T c}$ protocols. To achieve $50 \%$ of laboratory volume with $<9$-mSv dose, practices can implement several of the above options into their practice. $L D=$ low dose; HD = high dose; 1 $\mathrm{mCi}=37 \mathrm{MBq}$. additional dose reduction options for PET MPI but have not been as widely studied or implemented.

Stress-only PET MPI in 3D mode with myocardial blood flow assessment can be performed with less than a $1-\mathrm{mSv}$ radiation dose. Typical adult patients referred for PET MPI, however, are high-risk patients and are not always suited to stress-only imaging. In addition, coronary flow reserve (CFR), an emerging risk marker of coronary vascular dysfunction, cannot be estimated with stressonly imaging. However, one recent study using ${ }^{15} \mathrm{O}$-water MPI suggested that hyperemic myocardial blood flow may be more accurate than CFR for the diagnosis of obstructive epicardial CAD (accuracy, $86 \%$ vs. $78 \%$; $P<0.01)(53,54)$. On the other hand, another study suggested that although CFR and stress myocardial blood flow with ${ }^{82} \mathrm{Rb}$ provide powerful risk stratification, estimates of CFR may be more robust and less variable than stress myocardial blood flow (54). If stress myocardial blood flow is confirmed to be superior to CFR with the clinical PET perfusion tracers, stress-only PET MPI may be more widely implemented. Combining stress-only MPI with low-dose CT coronary angiography (55) (if CT results are abnormal) or with calcium score (56) can identify significant CAD that may warrant aggressive medical therapy. Despite the significant advantages of superior image quality, better detection of CAD, and lowradiation-dose imaging, PET MPI and PET/ CT MPI are not widely available, remain expensive, and are predominantly limited to pharmacologic stress (because exercise stress can be challenging with PET).

\section{RADIATION DOSE REDUCTION AFTER THE TEST}

Finally, several steps can be taken after completion of MPI to minimize the lifetime radiation dose. First, an accurate, clear, and unambiguous report and timely communication to the referring physician will reduce repeat, layered testing. Next, the MPI report should accurately list the administered radiotracer dose; soon, estimates 
of lifetime radiation exposure from medical imaging may become a requirement on the report. Periodic quality reviews of the laboratory for doses administered for MPI, image quality with lowdose MPI, and evaluation of the number of MPI procedures below the ASNC-recommended dose parameters of less than 9 $\mathrm{mSv}$ are suggested (22). Further, quality control of the scanners will optimize image quality and facilitate low-radiation-dose MPI. Last, staff radiation exposure should be followed according to ALARA (as low as reasonably achievable) principles, including rotation of duties for nuclear medicine technologists to avoid high radiation exposure to any individual technologist. A recent scientific statement on approaches to enhancing radiation safety in cardiovascular imaging (57) highlighted the need to educate referring physicians and health care providers on performing low-dose medical imaging and provided a list of online resources for radiation dose reduction from the various societies.

\section{CONCLUSION}

The rising barriers to radionuclide MPI from ionizing radiation and high costs mandate a change in the traditional practice of nuclear cardiology. The time to change is now. Novel imaging protocols and powerful imaging technologies have emerged to facilitate low-dose high-quality MPI. A secondary benefit of this change is that in the current challenging fiscal environment, medical facilities, practitioners, and practices can differentiate themselves by producing superior image quality at a faster pace and at a low dose. State-of-the-art nuclear cardiology practice requires embracing best practices for appropriate patient selection, patient-centered imaging protocols, use of novel protocols for traditional scanners, and adoption of laboratory practices to reduce lifetime radiation exposure for patients and staff members. The move to a lower radiation dose for MPI brings nuclear cardiology into the 21st century, spreading new best practices across the country and the world.

\section{REFERENCES}

1. Report to Congressional Requesters: Medicare Part B Imaging Services-Rapid Spending Growth and Shift to Physician Offices Indicate Need for CMS to Consider Additional Management Practices. Washington, DC: U.S. Government Accountability Office; 2008.

2. Einstein AJ. Effects of radiation exposure from cardiac imaging: how good are the data? J Am Coll Cardiol. 2012;59:553-565.

3. Hendel RC, Berman DS, Di Carli MF, et al. ACCF/ASNC/ACR/AHA/ASE/ SCCT/SCMR/SNM 2009 appropriate use criteria for cardiac radionuclide imaging: a report of the American College of Cardiology Foundation Appropriate Use Criteria Task Force, the American Society of Nuclear Cardiology, the American College of Radiology, the American Heart Association, the American Society of Echocardiography, the Society of Cardiovascular Computed Tomography, the Society for Cardiovascular Magnetic Resonance, and the Society of Nuclear Medicine. Circulation. 2009;119:e561-e587.

4. Shaw LJ, Iskandrian AE. Prognostic value of gated myocardial perfusion SPECT. J Nucl Cardiol. 2004;11:171-185.

5. Shaw LJ, Hage FG, Berman DS, Hachamovitch R, Iskandrian A. Prognosis in the era of comparative effectiveness research: where is nuclear cardiology now and where should it be? J Nucl Cardiol. 2012;19:1026-1043.

6. Shaw LJ, Hachamovitch R, Berman DS, et al. The economic consequences of available diagnostic and prognostic strategies for the evaluation of stable angina patients: an observational assessment of the value of precatheterization ischemia. Economics of Noninvasive Diagnosis (END) Multicenter Study Group. J Am Coll Cardiol. 1999;33:661-669.

7. Murthy VL, Naya M, Foster CR, et al. Improved cardiac risk assessment with noninvasive measures of coronary flow reserve. Circulation. 2011;124:2215-2224.

8. Naya M, Murthy VL, Taqueti VR, et al. Preserved coronary flow reserve effectively excludes high-risk coronary artery disease on angiography. J Nucl Med. 2014;55:248-255.
9. Garcia EV. Physical attributes, limitations, and future potential for PET and SPECT. J Nucl Cardiol. 2012;19(suppl 1):S19-S29.

10. Slomka PJ, Berman DS, Germano G. New cardiac cameras: single-photon emission CT and PET. Semin Nucl Med. 2014;44:232-251.

11. BIER VII: health risks from exposure to low levels of ionizing radiation. National Center for Healthy Housing website. http://www.nchh.org/Portals/0/ Contents/NAS_BEIR_VII_ReportinBrief_June2005.pdf. Published June 2005. Accessed March 2, 2015.

12. White paper: initiative to reduce unnecessary radiation exposure from medical imaging. U.S. Food and Drug Administration website. http://www.fda.gov/ Radiation-EmittingProducts/RadiationSafety/RadiationDoseReduction/ucm199994. htm. Updated February 5, 2015. Accessed March 2, 2015.

13. Dorbala S, Di Carli MF, Delbeke D, et al. SNMMI/ASNC/SCCT guideline for cardiac SPECT/CT and PET/CT 1.0. J Nucl Med. 2013;54:1485-1507.

14. Hendel RC, Cerqueira M, Douglas PS, et al. A multicenter assessment of the use of single-photon emission computed tomography myocardial perfusion imaging with appropriateness criteria. J Am Coll Cardiol. 2010;55:156-162.

15. American Society of Nuclear Cardiology: five things physicians and patients should question. Choosing Wisely website. http://www.choosingwisely.org/ doctor-patient-lists/american-society-of-nuclear-cardiology/. Published April 4, 2012. Accessed March 3, 2015.

16. Society of Nuclear Medicine and Molecular Imaging: five things physicians and patients should question. Choosing Wisely website. http://www.choosingwisely. org/doctor-patient-lists/society-of-nuclear-medicine-and-molecular-imaging/. Published February 21, 2013. Accessed March 3, 2015.

17. Gibbons RJ, Askew JW, Hodge D, Kaping B, Carryer DJ, Miller T. Appropriate use criteria for stress single-photon emission computed tomography sestamibi studies: a quality improvement project. Circulation. 2011;123:499-503.

18. Saifi S, Taylor AJ, Allen J, Hendel R. The use of a learning community and online evaluation of utilization for SPECT myocardial perfusion imaging. JACC Cardiovasc Imaging. 2013;6:823-829.

19. Lin FY, Dunning AM, Narula J, et al. Impact of an automated multimodality point-of-order decision support tool on rates of appropriate testing and clinical decision making for individuals with suspected coronary artery disease: a prospective multicenter study. J Am Coll Cardiol. 2013;62:308-316.

20. Burmahl B. New law mandates use of imaging appropriateness criteria. Radiological Society of North America website. http://rsna.org/NewsDetail. aspx?id=12360. Published June 1, 2014. Accessed March 3, 2015.

21. Congress passes legislation linking physician payments to appropriate use criteria. Society of Nuclear Medicine and Molecular Imaging website. http://www. snmmi.org/NewsPublications/NewsDetail.aspx?ItemNumber $=11205$. Published March 31, 2014. Accessed March 3, 2015.

22. Cerqueira MD, Allman KC, Ficaro EP, et al. Recommendations for reducing radiation exposure in myocardial perfusion imaging. J Nucl Cardiol. 2010; 17:709-718.

23. Depuey EG, Mahmarian JJ, Miller TD, et al. Patient-centered imaging. J Nucl Cardiol. 2012;19:185-215.

24. Duvall WL, Croft LB, Godiwala T, Ginsberg E, George T, Henzlova MJ. Reduced isotope dose with rapid SPECT MPI imaging: initial experience with a CZT SPECT camera. J Nucl Cardiol. 2010;17:1009-1014.

25. Marcassa C, Zoccarato O, Calza P, Campini R. Temporal evolution of administered activity in cardiac gated SPECT and patients' effective dose: analysis of an historical series. Eur J Nucl Med Mol Imaging. 2013;40:325-330.

26. Rozanski A, Gransar H, Hayes SW, et al. Temporal trends in the frequency of inducible myocardial ischemia during cardiac stress testing: 1991 to 2009. J Am Coll Cardiol. 2013;61:1054-1065.

27. Chang SM, Nabi F, Xu J, Raza U, Mahmarian JJ. Normal stress-only versus standard stress/rest myocardial perfusion imaging: similar patient mortality with reduced radiation exposure. J Am Coll Cardiol. 2010;55:221-230.

28. Duvall WL, Baber U, Levine EJ, Croft LB, Henzlova MJ. A model for the prediction of a successful stress-first Tc-99m SPECT MPI. J Nucl Cardiol. 2012;19:1124-1134.

29. Ferreira MJ, Cunha MJ, Albuquerque A, et al. Prognosis of normal stress-only gated-SPECT myocardial perfusion imaging: a single center study. Int J Cardiovasc Imaging. 2013;29:1639-1644.

30. Gemignani AS, Muhlebach SG, Abbott BG, Roye GD, Harrington DT, Arrighi JA. Stress-only or stress/rest myocardial perfusion imaging in patients undergoing evaluation for bariatric surgery. J Nucl Cardiol. 2011;18:886-892.

31. Gibson PB, Demus D, Noto R, Hudson W, Johnson LL. Low event rate for stressonly perfusion imaging in patients evaluated for chest pain. J Am Coll Cardiol. 2002;39:999-1004.

32. Heller GV, Bateman TM, Johnson LL, et al. Clinical value of attenuation correction in stress-only Tc-99m sestamibi SPECT imaging. J Nucl Cardiol. 2004;11:273-281. 
33. Kaminek M, Metelkova I, Budikova M, et al. Prognostic value of stress-only and stress-rest normal gated SPECT imaging: higher incidence of cardiac hard events in diabetic patients who underwent full stress-rest imaging. Biomed Pap Med Fac Univ Palacky Olomouc Czech Repub. May 30, 2014 [Epub ahead of print].

34. McMahon SR, Kikut J, Pinckney RG, Keating FK. Feasibility of stress only rubidium-82 PET myocardial perfusion imaging. J Nucl Cardiol. 2013; 20:1069-1075.

35. Duvall WL, Guma KA, Kamen J, et al. Reduction in occupational and patient radiation exposure from myocardial perfusion imaging: impact of stress-only imaging and high-efficiency SPECT camera technology. J Nucl Med. 2013; 54:1251-1257.

36. Shaw LJ, Mieres JH, Hendel RH, et al. Comparative effectiveness of exercise electrocardiography with or without myocardial perfusion single photon emission computed tomography in women with suspected coronary artery disease: results from the What Is the Optimal Method for Ischemia Evaluation in Women (WOMEN) trial. Circulation. 2011;124:1239-1249.

37. Mathur S, Heller GV, Bateman TM, et al. Clinical value of stress-only Tc-99m SPECT imaging: importance of attenuation correction. J Nucl Cardiol. 2013; 20:27-37.

38. Cervo M FC, Dorbala S, Spence D, Ding X, Bhattacharya M, Vija AH, Moore SC, Di Carli MF. Dose reduction in stress myocardial SPECT/CT using cardiac confocal collimation [abstract]. J Nucl Cardiol. 2014;21(suppl):769.

39. Garcia EV, Faber TL, Esteves FP. Cardiac dedicated ultrafast SPECT cameras: new designs and clinical implications. J Nucl Med. 2011;52:210-217.

40. DePuey EG. Advances in SPECT camera software and hardware: currently available and new on the horizon. J Nucl Cardiol. 2012;19:551-581.

41. Borges-Neto S, Pagnanelli RA, Shaw LK, et al. Clinical results of a novel wide beam reconstruction method for shortening scan time of Tc-99m cardiac SPECT perfusion studies. J Nucl Cardiol. 2007;14:555-565.

42. DePuey EG, Ata P, Wray R, Friedman M. Very low-activity stress/high-activity rest, single-day myocardial perfusion SPECT with a conventional sodium iodide camera and wide beam reconstruction processing. J Nucl Cardiol. 2012;19:931-944.

43. DePuey EG, Bommireddipalli S, Clark J, Thompson L, Srour Y. Wide beam reconstruction "quarter-time" gated myocardial perfusion SPECT functional imaging: a comparison to "full-time" ordered subset expectation maximum. J Nucl Cardiol. 2009;16:736-752.

44. Marcassa C, Campini R, Zoccarato O, Calza P. Wide beam reconstruction for halfdose or half-time cardiac gated SPECT acquisitions: optimization of resources and reduction in radiation exposure. Eur J Nucl Med Mol Imaging. 2011;38:499-508.

45. Madsen MT. Recent advances in SPECT imaging. J Nucl Med. 2007;48:661-673.

46. Sharir T, Slomka PJ, Hayes SW, et al. Multicenter trial of high-speed versus conventional single-photon emission computed tomography imaging: quantitative results of myocardial perfusion and left ventricular function. J Am Coll Cardiol. 2010;55:1965-1974.

47. Esteves FP, Raggi P, Folks RD, et al. Novel solid-state-detector dedicated cardiac camera for fast myocardial perfusion imaging: multicenter comparison with standard dual detector cameras. J Nucl Cardiol. 2009;16:927-934.
48. Einstein AJ, Blankstein R, Andrews H, et al. Comparison of image quality, myocardial perfusion, and left ventricular function between standard imaging and single-injection ultra-low-dose imaging using a high-efficiency SPECT camera: the MILLISIEVERT study. J Nucl Med. 2014;55:1430-1437.

49. Nakazato R, Berman DS, Hayes SW, et al. Myocardial perfusion imaging with a solid-state camera: simulation of a very low dose imaging protocol. $\mathrm{J} \mathrm{Nucl}$ Med. 2013;54:373-379.

50. Duvall WL, Sweeny JM, Croft LB, Ginsberg E, Guma KA, Henzlova MJ. Reduced stress dose with rapid acquisition CZT SPECT MPI in a non-obese clinical population: comparison to coronary angiography. J Nucl Cardiol. 2012;19:1927.

51. Esteves FP, Galt JR, Folks RD, Verdes L, Garcia EV. Diagnostic performance of low-dose rest/stress Tc-99m tetrofosmin myocardial perfusion SPECT using the 530c CZT camera: quantitative vs visual analysis. J Nucl Cardiol. 2014;21:158165 .

52. Gimelli A, Bottai M, Genovesi D, Giorgetti A, Di Martino F, Marzullo P. High diagnostic accuracy of low-dose gated-SPECT with solid-state ultrafast detectors: preliminary clinical results. Eur J Nucl Med Mol Imaging. 2012;39:83-90.

53. Danad I, Uusitalo V, Kero T, et al. Quantitative assessment of myocardial perfusion in the detection of significant coronary artery disease: cutoff values and diagnostic accuracy of quantitative $\left[{ }^{15} \mathrm{O} \mathrm{H}_{2} \mathrm{O}\right.$ PET imaging. $J$ Am Coll Cardiol. 2014;64:1464-1475.

54. Murthy VL, Lee BC, Sitek A, et al. Comparison and prognostic validation of multiple methods of quantification of myocardial blood flow with ${ }^{82}$ Rb PET. $J$ Nucl Med. 2014;55:1952-1958.

55. Pazhenkottil AP, Herzog BA, Husmann L, et al. Non-invasive assessment of coronary artery disease with CT coronary angiography and SPECT: a novel dose-saving fast-track algorithm. Eur J Nucl Med Mol Imaging. 2010;37:522527.

56. Uretsky S, Cohen R, Argulian E, et al. Combining stress-only myocardial perfusion imaging with coronary calcium scanning as a new paradigm for initial patient work-up: an exploratory analysis. J Nucl Cardiol. 2015;22:89-97.

57. Fazel R, Krumholz HM, Wang Y, et al. Exposure to low-dose ionizing radiation from medical imaging procedures. N Engl J Med. 2009;361:849-857.

58. Dilsizian V, Bacharach S, Beanlands R, et al. PET myocardial perfusion and metabolism clinical imaging. American Society of Nuclear Cardiology website. https://www.asnc.org/imageuploads/ImagingGuidelinesPETJuly2009.pdf. Published 2009. Accessed March 3, 2015.

59. Holly TA, Abbott BG, Al-Mallah M, et al. Single photon-emission computed tomography. J Nucl Cardiol. 2010;17:941-973.

60. Senthamizhchelvan S, Bravo PE, Esaias C, et al. Human biodistribution and radiation dosimetry of ${ }^{82} \mathrm{Rb}$. J Nucl Med. 2010;51:1592-1599.

61. Duvall WL, Sweeny JM, Croft LB, et al. Comparison of high efficiency CZT SPECT MPI to coronary angiography. J Nucl Cardiol. 2011;18:595-604.

62. Nkoulou R, Pazhenkottil AP, Kuest SM, et al. Semiconductor detectors allow low-dose-low-dose 1-day SPECT myocardial perfusion imaging. J Nucl Med. 2011;52:1204-1209. 